\title{
Transition from Galactic to Extragalactic Cosmic Rays
}

\author{
M. Kachelrie $\beta^{1}$ \\ ${ }^{1}$ Institutt for fysikk, NTNU, Trondheim, Norway
}

\begin{abstract}
The question at which energy the transition from Galactic to extragalactic cosmic rays takes place has been a long-standing conundrum in cosmic ray physics. In the past, the transition energy has been usually associated with one of the evident features of the cosmic ray spectrum: The second knee around $E \simeq 5 \times 10^{17} \mathrm{eV}$ or the ankle at $E \simeq 3 \times 10^{18} \mathrm{eV}$. I review anisotropy and composition data and show that they require that the transition from Galactic to extragalactic CRs has to happen early, at few $\times 10^{17} \mathrm{eV}$. As a result, a successful model for the transition should explain the ankle as a feature of the extragalactic CR spectrum. I review briefly such models and their implications.
\end{abstract}

\section{Introduction}

The question at which energy the transition from Galactic to extragalactic cosmic rays (CRs) takes place is one of the major unresolved issues of cosmic ray physics. Its answer is fundamental to our understanding of Galactic CR sources and the requirements on their acceleration mechanisms as well as the nuclear composition and the injection spectrum of extragalactic sources. In the past, the two most promising choices for the transition energy were to associate it with one of the evident features of the cosmic ray spectrum: The ankle at $E \simeq 3 \times 10^{18} \mathrm{eV}$ or the second knee around $E \simeq 5 \times 10^{17} \mathrm{eV}$. The former case offered a simple explanation for the sharpness of the ankle as the cross-over between the end of Galactic flux and the start of the extragalactic component. Moreover, it allowed for an extragalactic injection spectrum $Q(E) \sim 1 / E^{\alpha}$ with $\alpha \sim 2$, i.e. close to the theoretical expectation for diffusive shock acceleration. The main disadvantage of this suggestion is the enormous pressure it puts on acceleration models for Galactic CR sources. Moreover, this solution may lead to the following "coincidence problem": Since the acceleration and diffusion of CRs depends only on rigidity $\mathcal{R}=p / Z e$, one expects that the end of the Galactic CR spectrum shows a sequence of cut-offs at $Z E_{\max }$, the so-called Peters cycle. If the ankle is identified with the transition, it is natural to assume that the second knee corresponds to the iron knee. Thus, in this interpretation, the second knee signals the end of the Galactic iron flux from those sources which contribute the bulk of Galactic CRs. Therefore an additional Galactic population of CR sources would be required to fill the gap between the second knee and the ankle. If this population is unrelated to the standard population of Galactic CR sources, it is surprising that the normalisation of the two fluxes is so close.

The challenge to models identifying the second knee as the transition to extragalactic CRs is to find a physical mechanism which explains the ankle as a consequence of either the propagation of extragalactic CRs or of interactions in their sources. The first successful model of this kind explained the ankle by the dip in the pairproduction losses of protons on cosmic microwave background (CMB) photons, $p+\gamma_{\mathrm{CMB}} \rightarrow p+e^{+}+e^{-}[1]$. Only recently, photo-disintegration of $\mathrm{CR}$ nuclei on background photons inside their sources was suggested as a viable alternative mechanism $[2,3]$.

How can these two options for the transition energy, the second knee and the ankle, be experimentally distinguished? It is natural to expect that the nuclear composition of Galactic and extragalactic CRs should differ, because of propagation effects and of the different nature of their sources. In particular, the Galactic CR spectrum should become close to its end iron-dominated. A similar behaviour is expected for the extragalactic flux, shifted however to higher energies. Thus one expects the extragalactic composition at the transition energy to be lighter than the Galactic one. Therefore the signature of the transition in the composition is the disappearance of the (Galactic) iron, and the growth of a light extragalactic component. An additional powerful constraint comes from anisotropy measurements which combined with propagation studies of CRs in the Galactic magnetic field exclude a dominant light or intermediate Galactic component at high energies.

From a theoretical perspective, a successful model for the transition has to address both the extragalactic and the Galactic contributions to the measured fluxes of individual groups of elements, $F_{i}^{\mathrm{obs}}(E)=F_{i}^{\mathrm{exgal}}(E)+F_{i}^{\mathrm{gal}}(E)$, since only the combination is observed. We will start therefore this short review by a discussion of Galactic CRs at and above the knee in the next section. 


\section{The knee and the end of the Galactic CR spectrum}

Knowledge about the high-energy end of the Galactic CR spectrum and its nuclear composition is crucial in addressing the question of the transition to extragalactic CRs. A problem of particular importance is the question, if the second knee corresponds to the proton knee shifted by $Z=26$ in energy. Moreover, the question how strong the Galactic fluxes are suppressed at energies above $Z E_{\mathrm{k}}$ are important to determine theoretically the extragalactic fluxes in the transition region and below.

\subsection{Position of proton knee}

While there is a general agreement that the knee in the total CR spectrum at $E_{\mathrm{k}} \simeq 4 \mathrm{PeV}$ coincides with a suppression of the primary proton and/or helium flux, and that the composition becomes increasingly heavier in the energy range between the knee and $10^{17} \mathrm{eV}$ [4-7], there exist yet substantial uncertainties concerning the partial contributions of different mass groups to the primary CR composition. In particular, the question at which energy the suppression in the light components starts remains unclear. The results from KASCADE and KASCADE-Grande who as first experiments presented energy spectra for individual elemental groups suggested a proton knee around $2-5 \mathrm{PeV}$. The spectra for the heavier elements were consistent with a rigidity-dependent knee following $E_{\mathrm{k}}^{(Z)}=Z E_{\mathrm{k}}^{p}$ [7]. Since these results were obtained by measuring the number of electrons and muons on the ground, a rather large dependence on the used Monte Carlo simulations of strong interactions resulted. In particular, the relative fraction of light elements changed considerably between different simulations. Conflicting results for the knee in the flux of light elements were obtained by experiments using fluorescence technique and air shower arrays at high altitudes, as e.g. the ARGO-YBJ experiment [8]. This experiment combined results from its ground array with Cherenkov Telescope, finding a knee-like structure in the combined $\mathrm{p}+\mathrm{He}$ flux around $700 \mathrm{TeV}$.

Determining the knee energy in the flux of the light elements is of obvious importance: Since the combined $\mathrm{p}+\mathrm{He}$ flux is dominated by the He component at these energies, the ARGO-YBJ results would imply a proton knee at $E_{\mathrm{p}} \simeq 0.5 \mathrm{PeV}$ and an iron knee at $E_{\mathrm{Fe}} \simeq 20 \mathrm{PeV}$, respectively. If the source spectra would have an exponential cut-off at the rigidity $\mathcal{R} \simeq 0.5 \mathrm{PV}$, then the Galactic CR spectrum would end well below $0.1 \mathrm{EeV}$. This would require an additional Galactic component, even if the second knee corresponds to the transition energy. The alternative, lowering the transition energy even more, is problematic, because extragalactic CRs at such low energies may be hidden by magnetic horizons [9].

\subsection{Exponential cut-off, break or recovery?}

Most models for the knee assume ad-hoc how strong the flux is suppressed above the rigidity-dependent knee. If the suppression is associated with the maximal energy of an accelerator, one expects typically an exponential cutoff [10]. Then a second population of Galactic CR sources seems unavoidable, even if one associates the knee in the total spectrum with the one of helium, $E_{\mathrm{k}} \simeq E_{\mathrm{k}}^{\mathrm{He}} \simeq 4 \times$ $10^{15} \mathrm{eV}$.

In the original Hillas model [11], the flux has at $\mathcal{R}=$ $3 \times 10^{15} \mathrm{~V}$ instead of a cut-off only a break by $\Delta \beta=1.25$. Such a moderate steepening can be motivated e.g. by the analysis of Ref. [12]: Including strong field amplification as suggested by Bell and Lucek $[13,14]$ into a toy acceleration model, these authors found a break in the energy spectrum of accelerated protons, coinciding for typical values SNR parameters with the knee region. The strength $\Delta \beta$ of the steepening depends among others on the injection history, and in a typical test particle ansatz $\Delta \beta=0.9$ was found. For such a modest steepening, a second Galactic CR population can be avoided, as e.g. the model of Ref. [15] shows where Vela accounts for the Galactic flux between $200 \mathrm{TeV}$ and the second knee.

Another option is the escape model [16, 17] which connects the knee with a change in the propagation of Galactic CRs. For a coherence length $l_{\mathrm{c}} \simeq(2-5) \mathrm{pc}$ of the turbulent field and a relatively weak turbulent magnetic field, a knee-like structure at $E / Z=$ few $\times 10^{15} \mathrm{eV}$ was found, which is sufficiently strong to explain the proton knee observed by KASCADE. At higher energies, the proton flux recovers in this model as indicated by KASCADEGrande data. The resulting intensity of the four other elemental groups in this model are consistent with the energy spectra of CR nuclei determined by KASCADE and KASCADE-Grande up to the second knee.

\section{Anisotropy and composition}

\subsection{Anisotropy}

The observed distribution of CR arrival directions is highly isotropic, and thus an efficient mechanism for the isotropisation of the CR momenta exists. Agent of this isotropisation are turbulent magnetic fields, since charged CRs scatter efficiently with field modes which wavelength matches their Larmor radius. As a result, CRs perform on scales larger than the correlation length of the turbulent field a random walk, and the memory of the initial source location is mostly erased. Since large wavelengths of the turbulent field modes are less abundant, CRs with higher energy are scattered less efficiently. Therefore, the CR anisotropy should increase monotonically with energy. More precisely, if the turbulent field follows a Kolmogorov power law as suggested by the observed $\mathrm{B} / \mathrm{C}$ ratio, the dipole anisotropy $\delta$ should increase with energy as $\delta \propto E^{1 / 3}$. If the Larmor radius $R_{L}$ of a CR exceeds the coherence length of the magnetic field, one enters the small-angle scattering regime with $\delta \propto E^{2}$.

The (projected) dipole anisotropy $\delta$ measured by several experiments is shown in Fig. 1 as function of energy. Below $10^{17} \mathrm{eV}$, the phase of the anisotropy shown in the left panel is approximately constant, except for a flip at $\simeq 200 \mathrm{TeV}$. Such a behaviour is expected in 

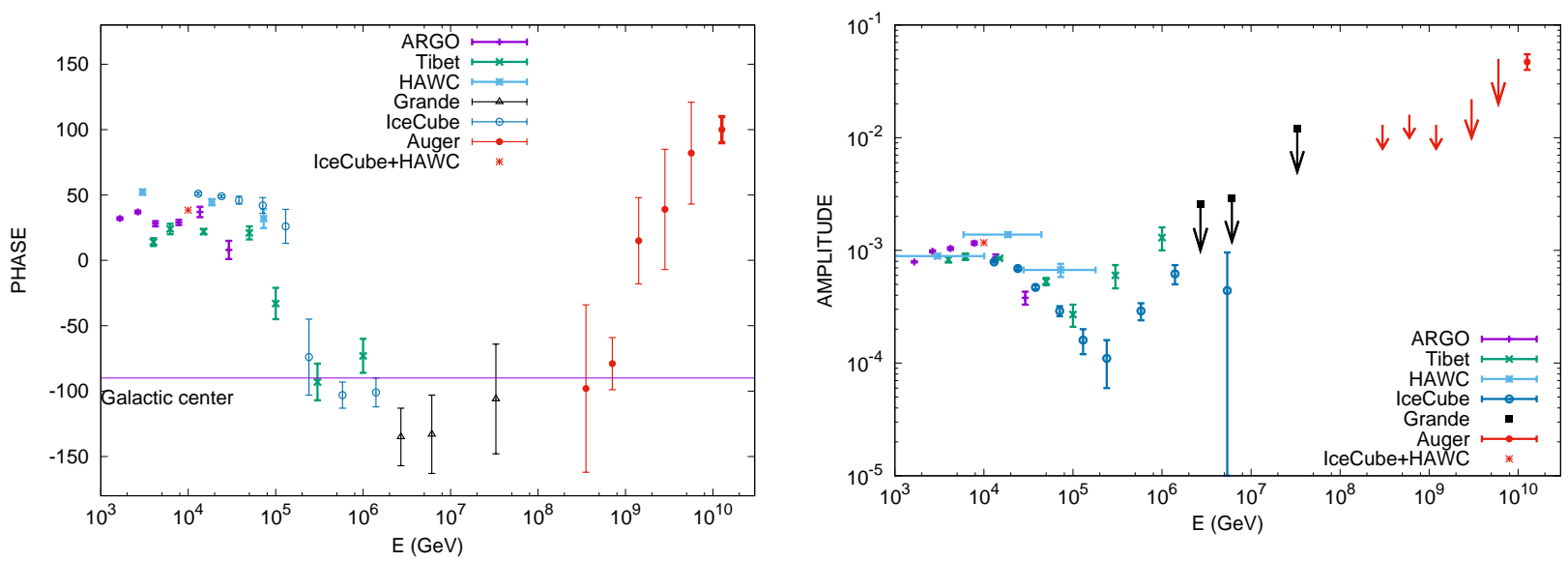

Figure 1. The dipole component of the CR anisotropy as function of energy: the phase (left panel) and the amplitude (right panel); adopted from Ref. [18].

the case of anisotropic CR diffusion: Then the projection effect on the local magnetic field line leads to flip of the phase, if below and above $200 \mathrm{TeV}$ two sources which are located in the opposite (relative to the local magnetic field line) hemisphere dominate the CR dipole anisotropy [19-21]. This interpretation is supported by the two plateaus in the dipole amplitude shown in the right panel, which are visible in the range $2-20 \mathrm{TeV}$ and (less clear) $500-10.000 \mathrm{TeV}$ : These are the consequence of the energy-independent contribution of two single sources to the dipole anisotropy.

Finally, at energies above $10^{17} \mathrm{eV}$, the phase changes smoothly towards 100 degrees in R.A., i.e. it points roughly towards the Galactic anticentre. In Ref. [22], the two-dimensional direction of the dipole was reconstructed also: The estimated direction may be connected to an overdensity in the local galaxy distribution, seen e.g. in the 2MRS catalogue [23]. Thus the behaviour of the dipole anisotropy suggests that the transition from Galactic to extragalactic CRs starts at $10^{17} \mathrm{eV}$.

\subsection{Composition}

Additionally to the all-particle CR spectrum above the knee, data on the primary composition have become available in the last decade: The KASCADE-Grande experiment measured the composition up to $2 \times 10^{17} \mathrm{eV}$ [7], while the Auger collaboration derived the fraction of four different elemental groups above $2 \times 10^{17} \mathrm{eV}$ [24]. These measurements can be summarized as follows: First, the proton fraction amounts to $\sim 30-100 \%$ in the energy range between $7 \times 10^{17} \mathrm{eV}$ and $3 \times 10^{18} \mathrm{eV}$ and decreases afterwards, while the fraction of intermediate nuclei increases. Second, the iron fraction in the energy range between $7 \times 10^{17} \mathrm{eV}$ and $2 \times 10^{19} \mathrm{eV}$ is consistent with zero and limited by $\lesssim 15-20 \%$. Going to lower energies, a nonzero iron fraction $\sim 20 \%$ appears in the lowest energy bin.

Despite both theoretical and experimental uncertainties, the following conclusions can be drawn: Using only the composition data, the iron fraction implies that the
Galactic contribution to the observed CR spectrum has to die out before $7 \times 10^{17} \mathrm{eV}$. The confidence in this conclusion is strengthened considerably, if one combines the composition and anisotropy measurements: In Ref. [25] it was shown that a light (intermediate) Galactic CR flux leads to a dipole of order $20 \%$ (10\%), overshooting clearly the limits which are on the percent level [22]. Thus the dominant light-intermediate contribution to the CR flux measured by the PAO above $3 \times 10^{17} \mathrm{eV}$ has to be extragalactic.

\section{Constraints from neutrino and photon secondaries}

High-energy cosmic rays can interact with gas or photons in their sources, and with photons from the extragalactic background light (EBL) during propagation. Any process involving hadronization leads mainly to the production of pions, and isospin symmetry fixes then the ratio of charged to neutral pions produced. The production of neutrinos is thus intimately tied to the one of photons, and both depend in turn on the flux of primary CRs. Thus the observation of these CR secondaries can provide important information on extragalactic CRs.

Let us recall first a few basic properties of the secondary photon and neutrino fluxes in hadronic interactions on nuclei and photons, respectively. Photons are mainly produced by $\pi^{0} \rightarrow 2 \gamma$, with an energy spectrum $d n / d E_{\gamma}=$ const. The maximal and minimal energy of these photons is $E_{\min }^{\max }=\frac{m_{\pi}}{2} \sqrt{\frac{1 \pm \beta}{1 \mp \beta}}$. The energy spectrum of the decay photons from pions with fixed energy plotted as function of $\ln \left(E_{\gamma}\right)$ corresponds therefore to a symmetric box around $m_{\pi}$, cf. Fig. 2. A signature of photons from hadronic interactions is thus the symmetry of the photon spectra with respect to the pion mass. The low threshold and the approximate Feynman scaling in hadronic interactions implies then $d N_{\gamma} / d E \sim d N_{C R} / d E$. By contrast, in p $\gamma$ interactions the threshold $E_{\mathrm{th}} \gtrsim m_{\pi} m_{p} / \varepsilon_{\gamma}$ with $\varepsilon_{\gamma}$ as the 

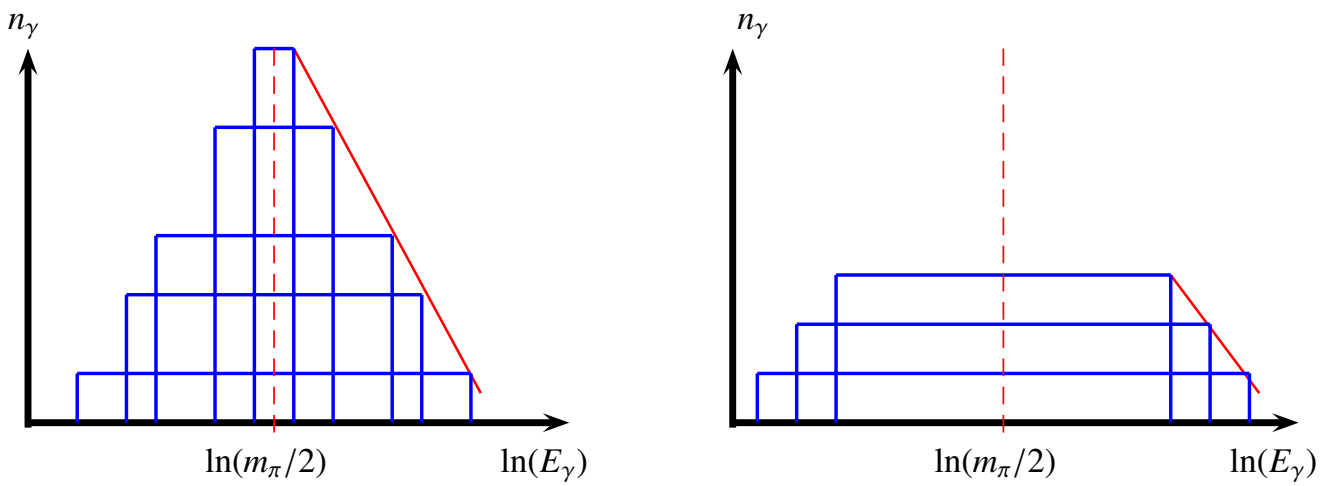

Figure 2. The photon spectrum $n_{\gamma}=\mathrm{d} N / \mathrm{d} E_{\gamma}$ produced in $\pi^{0}$ decays as function of $\ln \left(E_{\gamma}\right)$ for $p p$ (left panel) and for $p \gamma$ (right panel) interactions.

typical energy of the background photons leads to

$$
d N_{\gamma} / d E \sim \begin{cases}E^{-1} & \text { for } E<E_{\mathrm{th}}, \\ d N_{C R} / d E & \text { for } E>E_{\mathrm{th}} .\end{cases}
$$

Similar arguments hold for neutrino production. Cosmogenic neutrinos are mostly produced in interactions on EBL photons with energy $\varepsilon_{\gamma} \lesssim 10 \mathrm{eV}$. Taking into account that $\left\langle E_{v}\right\rangle=E_{p} / 20$, this implies that the flux of cosmogenic neutrinos is suppressed below $E \sim 2 \times 10^{17} \mathrm{eV}$. If neutrinos are produced by $\mathrm{p} \gamma$ interactions in the source, e.g. on radiation from an accretion disk with $\varepsilon_{\gamma} \lesssim 1 \mathrm{eV}$, one expects as threshold $E_{\mathrm{th}} \sim 2 \times 10^{18} \mathrm{eV}$. In contrast, $p p$ interactions lead to a neutrino flux without threshold.

\section{Diffuse extragalactic gamma-ray background and diffuse neutrino flux}

The Universe is opaque to the propagation of gamma-rays with energies in the TeV region and above. Such photons are absorbed by pair production on the EBL. As a result, the extragalactic photon flux at energies $E \gtrsim 1 \mathrm{TeV}$ is strongly attenuated. High-energy photons are however not really absorbed but initiate electromagnetic cascades, via the processes $\gamma+\gamma_{b} \rightarrow e^{+}+e^{-}$and $e^{ \pm}+\gamma_{b} \rightarrow e^{+-} \gamma$. The cascade develops very fast until it reaches the pair creation threshold. Thus the Universe acts as a calorimeter for electromagnetic radiation, accumulating it in the $\mathrm{MeV}-\mathrm{TeV}$ range as an extragalactic gamma-ray background (EGRB). The observed EGRB limits therefore all processes that inject electromagnetic energy. In Ref. [26], a measurement of the EGRB by Fermi-LAT was used to constrain strongly evolving UHECR models. In the meanwhile, the measurement of the EGRB was extended to higher energies [27] and, as a result, the limits on the allowed cascade radiation and the limit on the cosmogenic neutrino flux drop by a factor 3 to $\omega_{\text {casc }} \leq 2 \times 10^{7} \mathrm{eV} / \mathrm{cm}^{3}$. Moreover, the contribution from unresolved sources is now estimated to be as large as $50-80 \%$ [28]. Taking these results at face value, the room for any additional injection of photons is very limited. It is therefore desirable that the same source class explains both the UHECRs and the observed neutrino flux by IceCube.
The majority of neutrino sources is however rather weak, so that an identification of neutrino sources via neutrinos multipletts has failed so far ${ }^{1}$. Instead a diffuse flux of surprisingly large magnitude was discovered [29]: While its high-energy part is consistent with $\alpha \sim 2.1$ and a normalisation close to the cascade bound, at lower energies a softer component appears. The sources of this soft component has to be either extragalactic and hidden, or Galactic but close to isotropic.

\section{Models for extragalactic CRs}

The first model able to explain the ankle as a feature of the extragalactic CR spectrum was the dip model [1]. The main assumption of this model is that the extragalactic CR flux consists of protons, with a maximal admixture of $\lesssim 10 \%$ of helium. Then the ankle can be explained as a feature in the extragalactic CR spectrum imprinted by pair-production losses of protons on CMB photons. This elegant possibility has been however excluded by composition measurements, in particular of the PAO. It is interesting to note that meanwhile even the non-observation of cosmogenic neutrinos challenges this model [30].

Since composition measurements, in particular of the fluctuations of the shower maximum $\operatorname{RMS}\left(X_{\max }\right)$, pointed to an increase of mean mass number of CRs with increasing energy, models including nuclei were proposed. For instance, the models of [31-33] used a mixed composition together with a power-law in rigidity and an exponential cut-off, $Q(\mathcal{R})=Q_{i} \mathcal{R}^{-\alpha} \exp \left(-\mathcal{R} / \mathcal{R}_{\max }\right)$, for the injection spectrum. These models could reproduce $X_{\max }$ and $\operatorname{RMS}\left(X_{\max }\right)$ data, but lead to the ankle as transition energy. Thus such models require an additional light extragalactic component below the ankle. The spectral index of the injection spectrum of this additional population should be steeper $\left(Q(\mathcal{R}) \propto \mathcal{R}^{-2.7}\right)$ than the one of population responsible for the spectrum above the ankle [34]. Again, it is surprising that the normalisation of these two contributions is so close, if these two populations are unrelated.

\footnotetext{
${ }^{1}$ An exception may be the coincidence between the IceCube170922A event and the blazar TXS 0506+056
} 

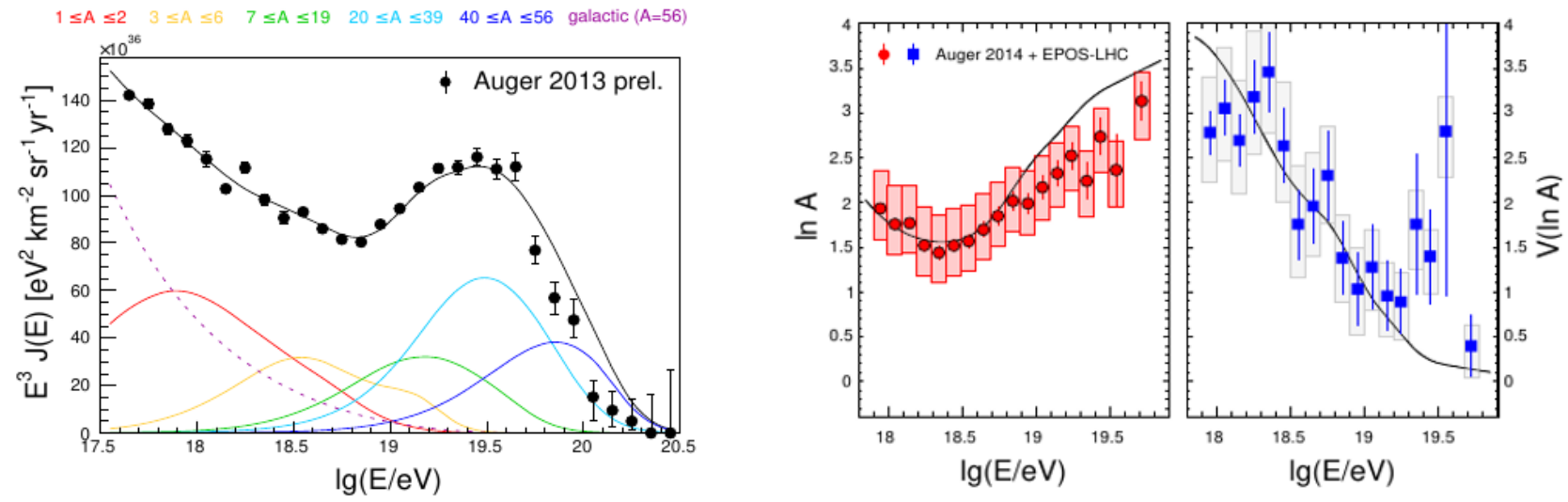

Figure 3. The energy spectrum, $X_{\max }$ and $\operatorname{RMS}\left(X_{\max }\right)$ as functions of energy for an injection composition following a Galactic mixture; from Ref. [3].

The end of the proton component measured by KASCADE-Grande can be extended smoothly to the one observed by the PAO by a power law with slope $\alpha \sim 2.2$. Any model aiming to extend the extragalactic flux below the ankle has to explain this proton component. In Ref. [35], it was assumed that this flux reflects the original injection spectrum of protons, since the slope is consistent with the one expected from diffusive shock acceleration. It was shown that star-forming galaxies cannot explain this proton component, while BL Lacs/FR I galaxies could both provide the proton component and a dominant contribution to the observed neutrino flux and the EGRB. However, this work did not address the question of heavier nuclei required by the composition measurements.

In an alternative scenario, the extragalactic proton component originates from the photo-disintegration of heavier nuclei in photon fields present in the source [2, 3]. This mechanism has been employed in the specific model of UHECR acceleration by gamma-ray bursts in Refs. [2, 36]. In this model, the photo-disintegration of low-energy nuclei leads to a steeping of their spectra from $\alpha \simeq 2.1-2.2$ to $\alpha \simeq 1$. Only the proton spectrum follows the original acceleration spectrum because of a decaying neutron component which escapes from the source. While the original model resulted in a transition at the ankle [2], choosing a stronger redshift evolution $\left(Q(z) \propto(1+z)^{3.5}\right)$ of GRBs increases the extragalactic contribution below the ankle [36]. Nevertheless, the required Galactic iron fraction is with, e.g., $20 \%$ at $10^{18} \mathrm{eV}$ relatively high compared to the determination from Ref. [24].

In Ref. [3], the same mechanism was employed in a toy-model which parameters were inspired by those of a core of an active galactic nuclei (AGN). In the fiducial model of [3], a hard injection spectrum, $\alpha=1$, with a moderate maximal energy $E_{\max }^{p}=3 \times 10^{18} \mathrm{eV}$ was used together with a photon background with typical energy $\varepsilon_{\gamma}=0.1 \mathrm{eV}$. A good fit to both the energy spectrum and the composition data was obtained injecting a single nuclear species with intermediate mass number like e.g. Si, cf. with Fig. 3. For a more natural mixed composition of the injected CRs, the agreement with the data deteriorated somewhat, cf. with Fig. 3. In particular, the transition energy moves somewhat to lower energies.

Both the GRB and AGN models of Refs. [3, 36] relied on interactions of nuclei with a photon background. As a result of the threshold effect described above, the resulting neutrino fluxes are therefore suppressed in the interesting $\mathrm{TeV}-\mathrm{PeV}$ range and cannot explain the observations of IceCube. In contrast, models leading to large neutrino fluxes in the $0.1-1 \mathrm{PeV}$ energy range use typically $p p$ interactions and primaries with $10-100 \mathrm{PeV}$ energies. Thus such models have no direct connection to the sources of UHECRs.

A possible way how a single source class can explain both the extragalactic CR flux, its nuclear composition and the observed neutrino flux in IceCube was suggested in Ref. [37]. The model presented there assumes that UHECRs are accelerated in the core of (a subclass of) AGNs. Subsequently, the CR nuclei diffuse first through a zone dominated by photo-hadronic interactions, before they escape into a second zone dominated by hadronic interactions with gas. In the first zone, the energy-dependence of the photo-disintegration rates and the escape times leads together with rigidity-dependent cut-off to a rather small energy window in which a single nuclear species is unsuppressed. The flat proton component is generated again by escaping neutrons. In the second zone, on larger scales, the escaping nuclei interaction on gas and produce thereby a neutrino flux which can give a substantial contribution to the flux observed by IceCube. In an alternative scenario, the photon background was neglected and only $A p$ were included.

The diffuse fluxes of five elemental groups computed in this model are shown on the left for only $A p$ interactions and on the right for $A \gamma$ and $A p$ interactions. In the case of only hadronic interactions, $\alpha=1.8, E_{\max }^{p}=3 \times 10^{18} \mathrm{eV}$ and BL Lac evolution is used, otherwise $\alpha=1.5, E_{\max }^{p}=$ $6 \times 10^{18} \mathrm{eV}, \tau^{p \gamma}=0.29$ at $10^{19} \mathrm{eV}$ and AGN evolution. The hadronic interaction depth is normalised as $\tau_{0}^{p p}=0.035$ at $10^{19} \mathrm{eV}$. The diffuse fluxes are compared to experimen- 

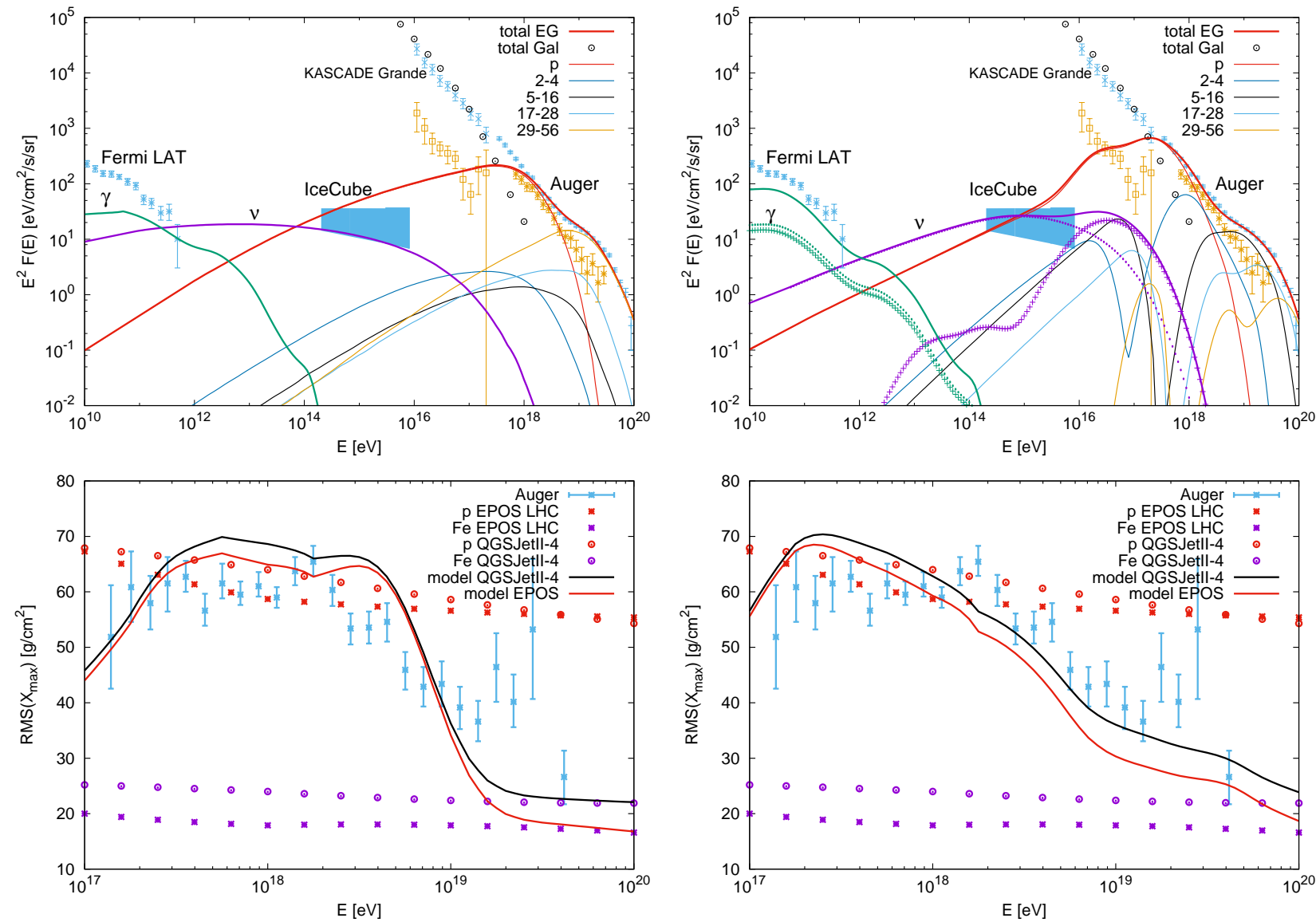

Figure 4. Diffuse flux (top) of five elemental groups and the resulting $\operatorname{RMS}\left(X_{\max }\right)$ (bottom) values; left for only hadronic, right with additional $A \gamma$ interactions [37].

tal results for the proton (orange error-bars) and the total flux from KASCADE, KASCADE-Grande (light-blue error-bars) [7] and Auger (dark-blue error-bars) [38, 39], the EGRB from Fermi-LAT (light-blue error-bars) [27], and the high-energy neutrino flux from IceCube (lightblue shaded area) [29] In both cases, the total CR flux including the ankle feature is well-fitted. Adding the Galactic CR flux determined in the escape model $[16,17]$ leads to a good description of the total flux in the transition region and below. Both cases lead to large neutrino fluxes, respecting at the same time however the EGRB limit. For illustration, the contribution of neutrinos and photons from $A \gamma$ and $A p$ interaction is shown on the right separately by crosses and dotted lines, respectively.

The lower panels compare the predictions of these two scenario for RMS $\left(X_{\max }\right)$ using the EPOS-LHC [40] and QGSJET-II-04 [41] models to data from Auger [42]. In the "hadronic only" scenario, insisting to reproduce the ankle requires a relatively low cut-off energy, and a small contribution of intermediate nuclei. This drives the composition towards a two-component model, consisting mainly of protons and iron. As a result, the composition data above $5 \times 10^{18} \mathrm{eV}$ are not well described. Since the spectra of intermediate $\mathrm{CNO}$ nuclei are cut off around the ankle, their contribution could be only increased if the proton flux is reduced. But a reduction of the proton flux would in turn reduce the neutrino flux and the model will fall short of explaining the IceCube data. In contrast, the scenario including photo-disintegration reproduces the composition data well, taking into account the systematic uncertainties.

Finally, let us comment briefly on some other recent extragalactic CR models which attempt to explain the diffuse neutrino flux observed by IceCube. The authors of Ref. [43] explain the IceCube neutrinos by CR interactions in the galaxy clusters surrounding UHECR sources. This scheme may be considered as a concrete physical model of the "only hadronic" scenario in Ref. [37]. The presented CR flux has however no ankle feature and requires therefore a second extragalactic component. The same limitation applies to the model of Ref. [44] which suggests lowluminosity GRB ankle as common source of UHECRs and neutrinos. The authors of Ref. [45] studied the central regions of active galaxies as sources of UHECRs. They followed the approach of Ref. [3] and concluded that low luminosity AGNs with no source evolution are favoured compared to models with strong source evolution or large photon backgrounds in the source.

\section{Conclusions}

Three pieces of evidence allow us to determine the energy at which the transition from Galactic to extragalactic CRs 
takes place. First, the behaviour of the dipole anisotropy suggests that the transition starts at $10^{17} \mathrm{eV}$. Second, the disappearance of the iron component at $5 \times 10^{17} \mathrm{eV}$ in the PAO composition data signals the end of the Galactic CR spectrum. Finally, combining the composition and anisotropy measurements, one can conclude that the dominant light-intermediate contribution to the CR flux measured above $7 \times 10^{17} \mathrm{eV}$ has to be extragalactic. Combining these different pieces of evidence, there is little room for doubt that the transition from Galactic to extragalactic CRs happens at the second knee, i.e. around $5 \times 10^{17} \mathrm{eV}$.

Using Occam's razor, one may exclude the possibility that the ankle is the cross-over of the fluxes of two independent extragalactic populations of UHECR sources. Then the ankle has to be explained as a consequence of either the propagation of extragalactic CRs or of interactions in their sources. The dip model which relied on the first possibility is excluded by composition measurements. A viable alternative uses photo-disintegration of $\mathrm{CR}$ nuclei on background photons inside their sources, which might be either AGN cores or GRBs. Successful models tend to favour unusual features as steep injection spectra or large fraction of injected intermediate nuclei which wait for a physical motivation. Requiring additionally that these UHECR sources explain the neutrino flux measured by IceCube poses another challenge. Developing concrete models which reproduce all the experimental data which have become available will be an important step towards understanding the sources of UHECRs.

\section{References}

[1] V. Berezinsky, A.Z. Gazizov, S.I. Grigorieva, Phys. Rev. D74, 043005 (2006)

[2] N. Globus, D. Allard, R. Mochkovitch, E. Parizot, Mon. Not. Roy. Astron. Soc. 451, 751 (2015)

[3] M. Unger, G.R. Farrar, L.A. Anchordoqui, Phys. Rev. D92, 123001 (2015)

[4] M. Aglietta et al. (EAS-TOP), Astropart. Phys. 21, 583 (2004)

[5] T. Antoni et al. (KASCADE), Astropart. Phys. 24, 1 (2005)

[6] R. Abbasi et al. (IceCube), Astropart. Phys. 42, 15 (2013)

[7] W.D. Apel et al., Astropart. Phys. 47, 54 (2013), 1306.6283

[8] P. Montini (ARGO-YBJ), Nucl. Part. Phys. Proc. 279-281, 7 (2016)

[9] E. Parizot, Nucl. Phys. Proc. Suppl. 136, 169 (2004)

[10] R.J. Protheroe, Astropart. Phys. 21, 415 (2004)

[11] A.M. Hillas, J. Phys. G31, R95 (2005)

[12] L.O. Drury, E. van der Swaluw, O. Carroll (2003), astro-ph/0309820

[13] A.R. Bell, S.G. Lucek, Mon. Not. Roy. Astron. Soc. 321, 433 (2001)

[14] A.R. Bell, Mon. Not. Roy. Astron. Soc. 353, 550 (2004)
[15] M. Bouyahiaoui, M. Kachelrieß, D.V. Semikoz (2018), 1812.03522

[16] G. Giacinti, M. Kachelrieß, D.V. Semikoz, Phys. Rev. D90, 041302 (2014)

[17] G. Giacinti, M. Kachelrieß, D.V. Semikoz, Phys. Rev. D91, 083009 (2015)

[18] M. Kachelrieß, D.V. Semikoz (2019), in preparation

[19] F.C. Jones, Astrophys. J. 361, 162 (1990)

[20] V. Savchenko, M. Kachelrieß, D.V. Semikoz, Astrophys. J. 809, L23 (2015)

[21] M. Ahlers, Phys. Rev. Lett. 117, 151103 (2016)

[22] A. Aab et al. (Pierre Auger), Astrophys. J. 868, 4 (2018)

[23] P. Erdogdu et al., Mon. Not. Roy. Astron. Soc. 368, 1515 (2006)

[24] M. Unger (Pierre Auger), this proceedings (2018)

[25] G. Giacinti, M. Kachelrieß, D.V. Semikoz, G. Sigl, JCAP 1207, 031 (2012)

[26] V. Berezinsky, A. Gazizov, M. Kachelrieß, S. Ostapchenko, Phys. Lett. B695, 13 (2011)

[27] M. Ackermann et al. (Fermi-LAT), Astrophys. J. 799, 86 (2015)

[28] M. Ackermann et al. (Fermi-LAT), Phys. Rev. Lett. 116, 151105 (2016)

[29] M.G. Aartsen et al. (IceCube), Astrophys. J. 833, 3 (2016)

[30] J. Heinze, D. Boncioli, M. Bustamante, W. Winter, Astrophys. J. 825, 122 (2016)

[31] D. Allard, E. Parizot, E. Khan, S. Goriely, A.V. Olinto, Astron. Astrophys. 443, L29 (2005)

[32] D. Allard, E. Parizot, A.V. Olinto, Astropart. Phys. 27, 61 (2007)

[33] D. Hooper, S. Sarkar, A.M. Taylor, Astropart. Phys. 27, 199 (2007)

[34] R. Aloisio, V. Berezinsky, P. Blasi, JCAP 1410, 020 (2014)

[35] G. Giacinti, M. Kachelrieß, O. Kalashev, A. Neronov, D.V. Semikoz, Phys. Rev. D92, 083016 (2015)

[36] N. Globus, D. Allard, E. Parizot, Phys. Rev. D92, 021302 (2015)

[37] M. Kachelrieß, O. Kalashev, S. Ostapchenko, D.V. Semikoz, Phys. Rev. D96, 083006 (2017)

[38] A. Letessier-Selvon et al. (Pierre Auger), Braz. J. Phys. 44, 560 (2014)

[39] A. Aab et al. (Pierre Auger), Phys. Rev. D90, 122006 (2014)

[40] T. Pierog, I. Karpenko, J.M. Katzy, E. Yatsenko, K. Werner, Phys. Rev. C92, 034906 (2015)

[41] S. Ostapchenko, Phys. Rev. D83, 014018 (2011)

[42] A. Aab et al. (Pierre Auger), Phys. Rev. D90, 122005 (2014)

[43] K. Fang, K. Murase, Nature Phys. 14, 396 (2018)

[44] D. Boncioli, D. Biehl, W. Winter (2018)

[45] A.D. Supanitsky, A. Cobos, A. Etchegoyen, Phys. Rev. D98, 103016 (2018) 